Vol. 19, $n^{\circ} 1 \mid 2015$

Varia

\title{
Bérard Jean, La justice en procès. Les mouvements de contestation face au système pénal (1968-1983)
}

Paris, Presses de Sciences Po, 2013, 296 pp., ISBN 9782724812721

Philippe Robert

\section{(2) OpenEdition}

\section{Journals}

Édition électronique

URL : http://journals.openedition.org/chs/1559

DOI : $10.4000 /$ chs. 1559

ISSN : 1663-4837

\section{Éditeur}

Librairie Droz

Édition imprimée

Date de publication : 1 juin 2015

Pagination : 119-122

ISBN : 978-2-600-01953-8

ISSN : 1422-0857

Référence électronique

Philippe Robert, «Bérard Jean, La justice en procès. Les mouvements de contestation face au système pénal (1968-1983) », Crime, Histoire \& Sociétés / Crime, History \& Societies [En ligne], Vol. 19, n¹ | 2015 mis en ligne le 01 juin 2017, consulté le 24 septembre 2020. URL : http://journals.openedition.org/chs/ 1559 ; DOI : https://doi.org/10.4000/chs.1559

Ce document a été généré automatiquement le 24 septembre 2020

(c) Droz 


\section{Bérard Jean, La justice en procès. Les mouvements de contestation face au système pénal (1968-1983)}

Paris, Presses de Sciences Po, 2013, 296 pp., ISBN 9782724812721

\section{Philippe Robert}

\section{RÉFÉRENCE}

Bérard Jean, La justice en procès. Les mouvements de contestation face au système pénal (1968-1983), Paris, Presses de Sciences Po, 2013, 296 pp., ISBN 9782724812721.

Jean

Bérard a publié dans la collection 'Histoire' des Presses de Sciences Po un ouvrage tiré de la thèse qu'il avait soutenue en 2010 sous la direction de Danièle Tartakowski. Le

titre

- La justice en procès - rend imparfaitement compte de la matière du livre : s'il peut convenir à la première partie dédiée à la politisation des questions pénales (au début des années 1970), il s'adapte difficilement à la seconde - l'éclatement des positions militantes en matière pénale (à la fin de la décennie) - où il est moins question de mettre la justice en procès que de la mobiliser à différentes fins. Finalement, le soustitre - Les mouvements de contestation face au système pénal (1968-1983) - explique mieux l'entreprise.

2 La position défendue par J. Bérard est simple : aux lendemains de mai 1968, la question pénale est devenue une cible de mouvements sociaux qui l'ont découverte à l'occasion de la répression appliquée à leurs militants et qui ont tenté de l'inclure dans une critique radicale reposant sur un schéma de lutte des classes. Cette première période a été marquée par des revendications de dépénalisation. Mais cette contestation s'est épuisée et les positions de plusieurs mouvements sociaux ont alors pivoté : ils ont cherché à obtenir, au contraire, des pénalisations nouvelles. 
3 Deux premiers chapitres sont dédiés aux velléités d'une critique radicale de la justice de classe et à sa rapide marginalisation. Que l'Extrême-Gauche - maoïste notamment cherche à inclure la justice de classe dans sa critique sociale globale ne cache pas la distance qui la sépare d'une contestation judiciaire - celle du Syndicat de la magistrature - beaucoup plus argumentée qui s'attache à mettre en lumière l'impunité de la criminalité financière et celle des infractions au droit du travail. Il en va de même, sur fond de révoltes pénitentiaires, entre la revendication abolitionniste du Comité d'action des prisonniers et les positions seulement réductionnistes adoptées par la contestation interne au système pénal ${ }^{1}$.

4 Mais ce sont les revendications féministes et homosexuelles de dépénalisation qui constituent l'essentiel des illustrations de la première partie, avec notamment le mouvement finalement assez rapide qui conduit du procès de Bobigny à la loi Veil sur l'interruption volontaire de grossesse.

5 La deuxième partie s'ouvre avec les mêmes acteurs, les mouvements militants féministes et homosexuels, mais dans des partitions différentes : pour les premiers, il s'agit cette fois d'obtenir, non plus une dépénalisation (de l'avortement), mais un durcissement de la répression ( $\mathrm{du}$ viol). Quant aux seconds, la revendication de dépénalisation des relations homosexuelles avec des mineurs consentants viendra se heurter à un mouvement de sens inverse de raidissement de la répression de toute forme de pédophilie envers des mineurs de 15 ans. Pour faire bonne mesure, un ultime chapitre traite de la pénalisation du racisme et des discriminations, ce qui conduit l'auteur à conclure que les mouvements sociaux sont passés d'une revendication de dépénalisation à une autre de criminalisation.

6 Certes, on connaît les débats et les divisions des mouvements sociaux sur le recours au pénal, notamment parmi les militantes féministes à propos du viol. Pareil constat constitue-t-il pour autant un analyseur suffisant? On pourrait lire la séquence dans d'autres termes: dans un premier temps, des mouvements représentant des groupes antérieurement minorisés ${ }^{2}$ cherchent à se débarrasser d'une menace pénale qui leur paraît viser spécifiquement leur groupe social; dans un second temps, ils cherchent à mobiliser l'arme pénale contre des périls qui les concernent particulièrement. Il n'y a pas nécessairement contradiction entre les deux moments parce que les cibles ne sont pas les mêmes : on cherche d'abord à écarter une incrimination menaçante, ensuite à en brandir une autre qui soit protectrice. Le couple dépénalisation de l'avortement / criminalisation du viol correspond bien à cette grille de lecture. Pour le mouvement homosexuel, la dépénalisation n'a pu aller jusqu'au bout de sa revendication initiale : elle s'est heurtée à un mouvement plus puissant de lutte contre la pédophilie. La stratégie de dé-minorisation a donc dû $\hat{u}^{3}$ passer sur un autre registre, civil cette fois et non plus pénal, la revendication du droit au mariage pour des personnes de même sexe. L'arène pénale peut être lue comme un champ qu'investissent différents acteurs collectifs qui tentent de se protéger contre une criminalisation, mais aussi éventuellement de se protéger par une criminalisation. L'analyse de toutes ces entreprises de pénalisation ou de dépénalisation gagnerait à utiliser les acquis des recherches sur la création de la loi (pénale) ${ }^{4}$.

7 Parmi tous ces acteurs, J. Bérard s'attache à ce que l'on pourrait taxer de mouvements sociaux. De manière curieuse, il ne se réfère pourtant pas le moindrement que ce soit aux travaux d'Alain Touraine et de ses disciples ${ }^{5}$. Pourtant, il ne semble pas se ranger parmi les historiens rétifs à la consultation des recherches sociologiques ${ }^{6}$. Quoiqu'il en 
soit, il n'est pas certain qu'il soit facile d'appliquer la même grille de lecture à un mouvement qui concerne 'plus de la moitié de l'humanité' et à un autre qui en réunit une fraction quantitativement beaucoup moins large ${ }^{7}$. Il n'est pas non plus certain qu'on puisse analyser de la même manière un mouvement qui parle pour soi (féministe ou homosexuel par exemple) et un autre qui parle pour autrui (les prisonniers, les victimes de discrimination, de racisme...) ; dans le premier cas, le militant est membre du groupe dont il défend la cause, pas nécessairement dans le second. Les capitaux que l'on mobilise, les scènes sur lesquelles on s'active, les stratégies que l'on développe peuvent varier beaucoup d'un cas à l'autre. Pour le second cas de figure, il serait probablement fécond d'emprunter aussi à la littérature sur les entrepreneurs de morale (moral entrepreneurs ${ }^{8}$ ).

8 Quoiqu'il en soit des promoteurs de ces entreprises de pénalisation et de dépénalisation, reste que le champ pénal - plus largement celui de la loi étatique - est dominé par le monde politique ${ }^{9}$. C'est ici qu'apparaît un autre aspect de l'ouvrage sous examen : son auteur voit dans le passage d'une critique de la répression pénale à son usage la source de la difficulté qu'aurait la Gauche à construire une doctrine cohérente et autonome en matière de sécurité. Mais le monde politique de Gauche ne se résume pas aux mouvements sociaux dont J. Bérard analyse l'action ${ }^{10}$. Comment soutenir alors que la politique publique de sécurité de la Gauche soit tellement dépendante de leurs revendications? L'auteur esquisse une explication p.15: on aurait considéré les mesures concédées à ces mouvements comme seulement symboliques, extérieurs donc à la répression pénale ordinaire. Mais lui-même reconnaît plus loin que la nouvelle loi sur le viol ne s'est pas limitée à un effet symbolique: antérieurement peuplée de voleurs, la prison l'est désormais de violeurs, non pas qu'ils soient aussi nombreux mais parce qu'ils y restent bien plus longtemps ${ }^{11}$. Par ailleurs, s'il s'agissait de mesures seulement symboliques, on ne comprendrait pas qu'elles aient empêché la mise au point d'une doctrine de Gauche autonome sur la sécurité. Au début de la deuxième partie, l'auteur met en cause le ralliement de fait de la Gauche au libéralisme politique qui l'aurait mise dans l'incapacité de se démarquer de la Droite en matière de sécurité. L'argument est trop peu précis pour être réellement explicatif.

9 Au milieu des années 1970, la Droite - qui est au pouvoir depuis les débuts de la V République mais qui sent son hégémonie menacée-

découvre enfin le moyen efficace pour endiguer le mouvement de remise en cause né de mai 1968 : au début de la décennie, les années Marcellin étaient hypnotisées sur le risque d'importation maoïste d'une subversion terroriste externe ; rien ne venant, le nouveau titulaire de la place Beauvau va recentrer l'alarme sur l'insécurité générée par la délinquance ordinaire

${ }^{12}$. Il faudra attendre la fin de la décennie pour qu'Alain Peyrefitte donne forme à cette doctrine par la loi Sécurité et libertés qui propose de négliger et l'explosion des vols ${ }^{13}$ et, bien entendu, la criminalité financière, et de se concentrer uniquement sur la violence. Comme personne n'en voit alors de manifestation patente, la Gauche, enfin parvenue au pouvoir, réussira à renvoyer partiellement cette loi aux oubliettes. Il n'empêche qu'elle a fixé jusqu'à nos jours la feuille de route de la Droite en matière de sécurité.

10 La Gauche, elle, s'est bornée dans le second lustre de la décennie 1970 à dénoncer un usage démagogique de l'alarme à l'insécurité. Arrivée aux affaires, elle tentera, avec le rapport Bonnemaison ${ }^{14}$, de forger une nouvelle doctrine de la sécurité. Faute d'un réel 
intérêt des sommets de l'État, il ne sera jamais possible de mettre en cohérence les volets répressif, préventif et de politique sociale. À la fin des années 1980, elle s'y essaiera à nouveau en cherchant à combiner la conversion d'une police d'ordre en police de proximité, le développement d'un néo-prolétariat de sécurité constitué d'agents locaux de médiation sociale financés sur des emplois-jeunes, enfin une politique destinée à sanctionner les communes refusant d'investir dans l'habitat social. L'ensemble aurait pu constituer l'armature d'une politique de sécurité réunifiée. Toutefois, ces mesures - engagées sur des bases incertaines et sans pilotage politique unifié - ont cristallisé de farouches oppositions politiques et corporatistes; elles n'auront pas le temps de prospérer, le retour de balancier politique de 2002 a entraîné leur ruine.

11 Au total, tenter d'en faire la matrice de l'histoire autrement complexe des politiques actuelles de sécurité n'ajoute pas grand-chose à l'intérêt que l'on prend à l'analyse des relations des mouvements sociaux des années 1970 à la justice pénale.

\section{BIBLIOGRAPHIE}

Becker, H., Outsiders, Studies in the Sociology of Deviance, NY, The Free Press of Glencoe, 1963.

Commission des maires sur la sécurité, Face à la délinquance : prévention, répression, solidarité, Paris, La documentation française, 1982.

Lascoumes, P., Les compromis parlementaires, combinaisons de surpolitisation et de souspolitisation, Revue française de science politique, 2009, 59, 3, pp. 455-478.

Neveu, E., Sociologie des mouvements sociaux, Paris, La Découverte, 1996.

Robert, Ph., Sociologie du crime, Paris, LaDécouverte, 2005.

Robert, Ph., The French Criminal Justice System, in Ruggiero, V., Ryan, M. (Eds), Punishment in Europe, A Critical Anatomy of Penal Systems, Houndmills, Basingstoke, Palgrave McMillan, 2013, pp. 111-131.

\section{NOTES}

1. ... sans oublier que les positions fermement corporatistes du syndicalisme pénitentiaire ont permis à l'administration de combiner préservation de l'ordre et mesures (au moins temporaires) de libéralisation.

2. Quoique pas toujours (démographiquement) minoritaires, ainsi dans le cas du mouvement féministe.

3. Après une période où l'on investit surtout la pénalisation des discriminations.

4. Synthèse in Robert, 2005, pp. 39-55 et ref. cit.

5. Non plus, soit dit en passant, qu'au Repères publié en 1996 par Érik Neveu.

6. Sa bibliographie contient même une publication de concert avec un sociologue des prisons.

7. Même s'il peut être surreprésenté dans le monde médiatique et politique. 
8. Becker, 1963.

9. Et par les juges et d'autres professionnels du champ.

10. Même si à la p. 18, on passe subrepticement des forces politiques - notamment des partis aux mouvements de contestation.

11. Robert, 2013, passim. Pour autant dater de cette loi le commencement d'une séquence d'inflation carcérale paraît exagéré. Pierre Lascoumes (2009) a ainsi pointé l'importance du troc conclu entre le gouvernement de Gauche du début des années 1990 et les sénateurs de Droite : laisser passer la réforme du code pénal contre une élévation considérable de l'échelle des peines. 12. On imagine souvent que la Droite s'est ralliée aux positions sécuritaires de l'Extrême-Droite. Chronologiquement, l'investissement politique de la sécurité est le fait de celle-là à un moment où celle-ci ne tient encore aucune place significative dans la vie politique. C'est seulement à partir des municipales de 1983 que l'Extrême-Droite récupèrera et radicalisera la revendication sécuritaire.

13. Que la police ne traite de toute façon tendanciellement plus.

14. Commission des maires, 1983. 